\title{
Efficacy of low dose temozolomide in combination with bortezomib in U87 glioma cells: a flow cytometric analysis
}

\author{
Panagiotis J. Vlachostergios, Christos N. Papandreou
}

Department of Medical Oncology, Faculty of Medicine, University of Thessaly, University Hospital of Larissa, Biopolis, Larissa, Greece

Submitted: 4 February 2013

Accepted: 30 March 2013

Arch Med Sci 2015; 11, 2: 307-310

DOI: 10.5114/aoms.2013.36919

Copyright $\odot 2015$ Termedia \& Banach

\section{Corresponding author:}

Panagiotis J. Vlachostergios MD

Department

of Medical Oncology

University of Thessaly School

of Health Sciences

Faculty of Medicine

Biopolis 41110, Larissa

Greece

Phone: +30241350 2785

E-mail: pvlacho@med.uth.gr

\section{Abstract}

Introduction: Maximizing responses of malignant gliomas is hampered by resistance to temozolomide (TMZ). Increasing efficacy but not toxicity is a key issue when testing drug combinations. The antimyeloma agent bortezomib (BZ) has shown promising results in vitro and is currently being tested in glioblastoma (GBM) patients. In this study we investigate whether reduction of TMZ dosage is feasible without compromising the antitumor effect of TMZ-BZ combination. Material and methods: U87 GBM cells were treated with increasing doses of $\operatorname{TMZ}(1,10,100,1000 \mu \mathrm{M}), \mathrm{BZ}(0.001,0.01,0.1,1)$ and the combination during a 48-hour period, and apoptotic or/and necrotic cell death was evaluated by flow cytometry.

Results: Bortezomib alone at a dose as low as $0.001 \mu \mathrm{M}$ markedly induced cell death, particularly late apoptosis, to a level which was comparable with high $\mathrm{TMZ}$ dosage. For combination treatments, the dose of $0.1 \mu \mathrm{M} \mathrm{BZ}$, which was more potent than the maximal dose of TMZ $(1000 \mu \mathrm{M})$, was chosen to be added to increasing $T M Z$ concentrations. The combination of $0.1 \mathrm{BZ} \mu \mathrm{M}$ BZ with low doses of TMZ $(1,10 \mu \mathrm{M})$ further increased the cell death rate in an additive manner, at levels higher than those induced by high doses of TMZ monotherapy $(100,1000 \mu \mathrm{M})$.

Conclusions: Efficacy of TMZ-BZ combination is feasible with low doses of TMZ in vitro.

Key words: temozolomide, bortezomib, glioma, U87, flow cytometry.

\section{Introduction}

Temozolomide (TMZ) with radiotherapy is the current standard of care for newly diagnosed glioblastoma multiforme (GBM), based on the randomized phase III EORTC-NCIC trial of Stupp et al. [1] However, improvement in overall survival of such patients was marginal and prognosis remains poor due to the high resistance of these tumors to treatment. Alternative dosing regimens have been tested for TMZ, in an effort to overcome repletion of 06-methylguanine methyltransferase (MGMT), which is considered to be the major mechanism of resistance to TMZ [2]. Prolonged administration of low doses of TMZ was shown to deplete MGMT activity in peripheral blood mononuclear cells, at the cost of increased hematologic toxicity in many cases [2]. Dose-limiting myelotoxicity was also observed when combinations of TMZ with MGMT inhibitors were tested with the aim of inducing chemo- and/or radio-sensitization [3]. 
Emerging evidence on the role of the proteasome in orchestration of DNA repair and on the antitumor activity of the proteasome inhibitor bortezomib (BZ) in GBM cell lines has led to the design of clinical trials evaluating the combination of this agent, already in clinical use for the treatment of myeloma, with TMZ [4-8]. However, existing preclinical data on the combination of proteasome inhibitors with TMZ in terms of chemosensitization and/or radiosensitization of gliomas are contradictory $[9,10]$.

In this work, we studied the cytotoxic effect of this combination in U87 GBM cells by flow cytometry, with the aim of deciphering whether BZ addition might magnify the cell death effect of lower doses of TMZ.

\section{Material and methods}

\section{Cell culture and reagents}

The human glioblastoma cell line U87 was purchased from the European Collection of Animal Cell Cultures (ECACC, UK) and all experiments were performed on cells between passage number 5 and 10, within 6 months of purchase. The cell line was cultured in Dulbecco's modified Eagle's medium (GIBCO, UK) supplemented with $10 \%$ heat-inactivated FBS (GIBCO, UK), 5\% L-glutamine (GIBCO, UK) and $1 \%$ penicillin-streptomycin (Euroclone, UK) at $37^{\circ} \mathrm{C}$ in a humidified $5 \% \mathrm{CO}_{2}$ atm. Temozolomide (TMZ) was purchased from Merck, UK. Bortezomib (BZ) was purchased from Janssen-Cilag Pharmaceuticals, Greece.

\section{Flow cytometry}

To study induction of apoptosis and/or necrosis, annexin V assays (TACS Annexin V-FITC Apoptosis Detection Kit, R\&D Systems Europe, UK) were performed according to the manufacturer's instructions. Briefly, $10^{6}$ cells were allowed to attach for $24 \mathrm{~h}$ at $37^{\circ} \mathrm{C}$ and were then either left untreated or were treated with $\mathrm{TMZ}$ for $48 \mathrm{~h}$ at ten-fold serial dilution concentrations between 1 and $10^{3} \mu \mathrm{M}$, or were treated with $\mathrm{BZ}$ at ten-fold serial dilution concentrations between $10^{-9}$ and $10^{-6} \mathrm{M}$. Bortezomib at a dose of $10^{-7} \mathrm{M}$ was chosen for co-administration with $T M Z$ at the above-mentioned doses and time. After incubations, treated and untreated cells were trypsinized and washed with $1 \times$ PBS. For each sample of $1 \times 10^{5}$ cells, $100 \mu$ l of Annexin Incubation Reagent were prepared, containing $10 \mu \mathrm{l}$ of $10 \mathrm{X}$ Binding Buffer, $10 \mu \mathrm{l}$ of propidium iodide $(\mathrm{PI})$, $1 \mu \mathrm{l}$ of Annexin V-FITC and $79 \mu \mathrm{l}$ of deionized $\mathrm{H}_{2} \mathrm{O}$. Cells were resuspended in the Annexin Incubation Reagent and incubated in the dark for $15 \mathrm{~min}$ at room temperature. Then, $400 \mu \mathrm{l}$ of $1 \times$ Binding Buffer were added and samples were analyzed by an EPICS XL-MCL Counter (Beckman Coulter, USA). Data were acquired from 50,000-100,000 cells (events) and were analyzed with Cell Quest software. Two independent experiments were carried out in duplicate and representative results are shown $(p<0.01)$.

\section{Synergism quotient calculation}

The synergism quotient was calculated by subtracting baseline values from all treatments and then dividing effects of combined treatments by the sum of individual treatments. A synergism quotient greater than 1.0 indicates that there is synergism for a given measured response [11].

\section{Results}

$98.12 \pm 0.26 \%$ of untreated U87 cells were alive after $48 \mathrm{~h}$. When $1 \mu \mathrm{M} \mathrm{TMZ}$ was administered, increased cell death began to occur, primarily through necrosis, whereas both early (Annexin+/PI-) and late apoptosis (Annexin+/PI+) were observed with higher doses of TMZ. Bortezomib alone at a dose as low as $0.001 \mu \mathrm{M}$ markedly induced cell death, particularly late apoptosis, to a level which was comparable with high TMZ dosage. Thus, BZ is more cytotoxic compared to TMZ for U87 cells. Increasing doses of BZ induced even greater apoptosis. For combination treatments, the dose of $0.1 \mu \mathrm{M} B Z$, which was more potent than the maximal dose of TMZ $\left(10^{3} \mu M\right)$, was chosen to be added to increasing $T M Z$ concentrations. The combination of BZ with low doses of TMZ further increased the cell death rate, particularly late apoptosis, leaving approximately $50 \%$ of the cell population alive (Figure 1). For the given doses and times, the interaction between the two drugs was considered to be additive, regarding apoptosis, necrosis or both (Table I).

\section{Discussion}

The combination of concurrent TMZ and radiotherapy with BZ in high grade gliomas is feasible in the clinical setting and produces tolerable toxicity [8]. Preclinical data on the interaction of the two drugs and type of cell death they induce together are limited. Therefore, we performed a FACS analysis of each drug alone and of the combination, evaluating apoptotic and/or necrotic events. Given previous data by others and our group on the use of $0.1 \mu \mathrm{M} \mathrm{BZ}$ as an inducer of apoptosis through several mechanisms, including PARP, Bcl-2, Bcl-xl, JNK/c-jun, NF- $\kappa B$, TRAIL, and MGMT, we specifically focused on this schedule $[6,7]$. Indeed, we found an additive effect on induction of cell death, mostly involving increased late apoptosis compared to either drug alone, which was evident even with the lowest dose of $\mathrm{TMZ}$ used.

A previous preclinical study on the antitumor efficacy of the new generation of proteasome inhibitor NPI-0052 as a single agent and in combina- 
TMZ $1 \mu \mathrm{M}$

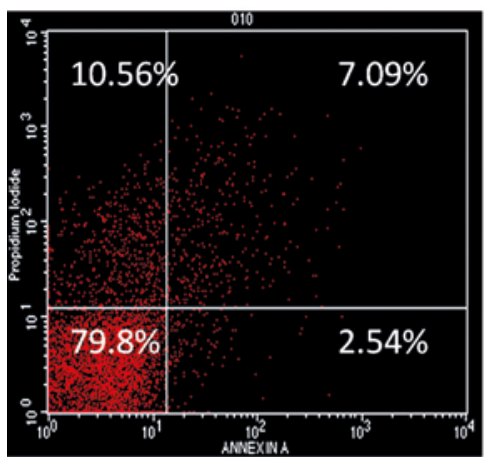

TMZ $10 \mu \mathrm{M}$

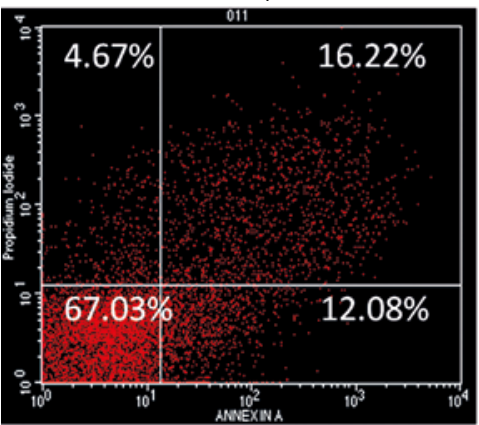

$\mathrm{TMZ} 100 \mu \mathrm{M}$

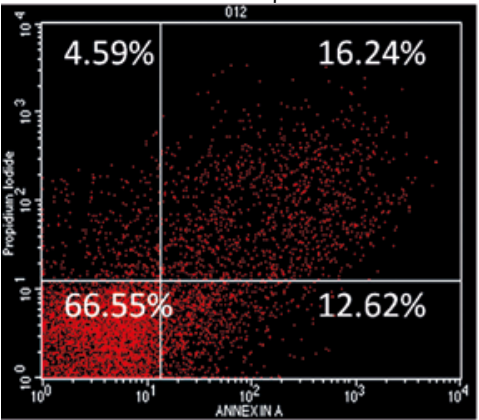

TMZ $1000 \mu \mathrm{M}$

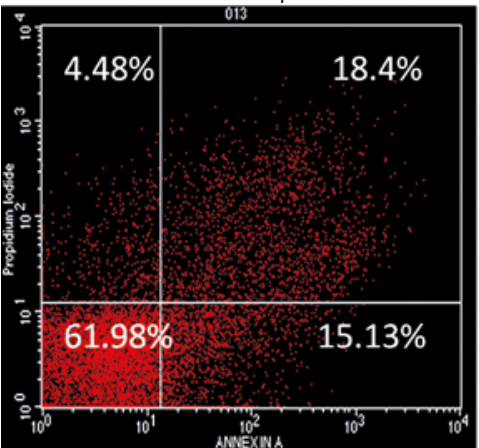

BZ $0.001 \mu \mathrm{M}$

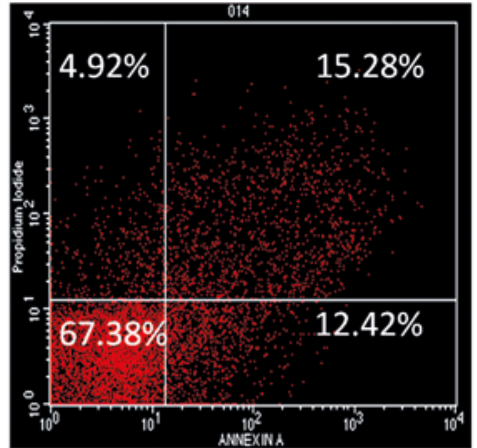

BZ $0.01 \mu \mathrm{M}$

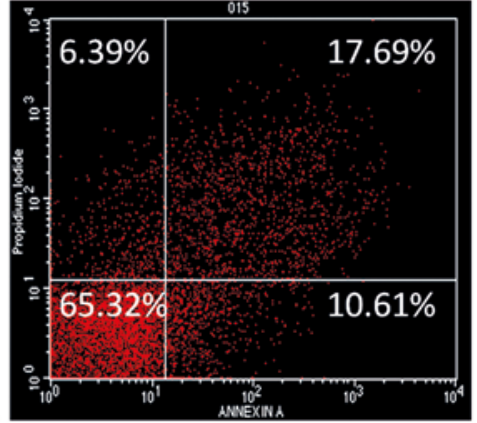

BZ $0.1 \mu \mathrm{M}$

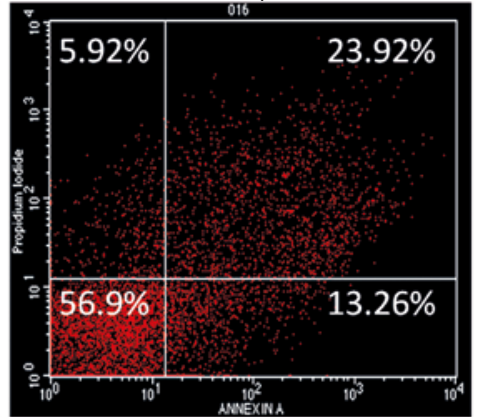

BZ $1 \mu M$

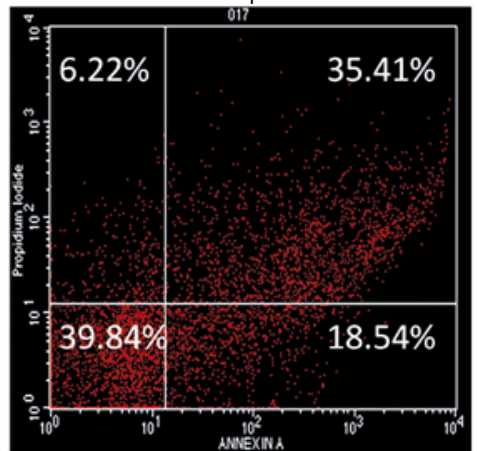

BZ $0.1 \mu \mathrm{M}+\mathrm{TMZ} 1 \mu \mathrm{M}$

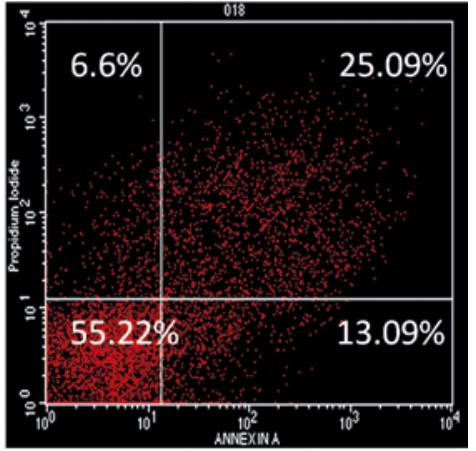

BZ $0.1 \mu M+T M Z 10 \mu M$

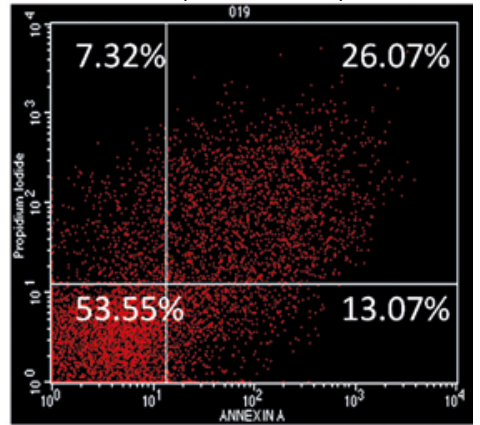

BZ $0.1 \mu M+T M Z 100 \mu M$

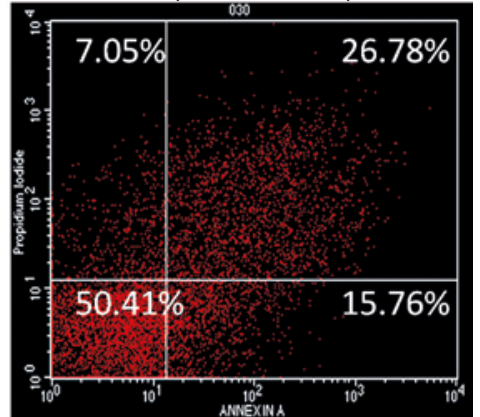

BZ $0.1 \mu \mathrm{M}+\mathrm{TMZ} 1000 \mu \mathrm{M}$

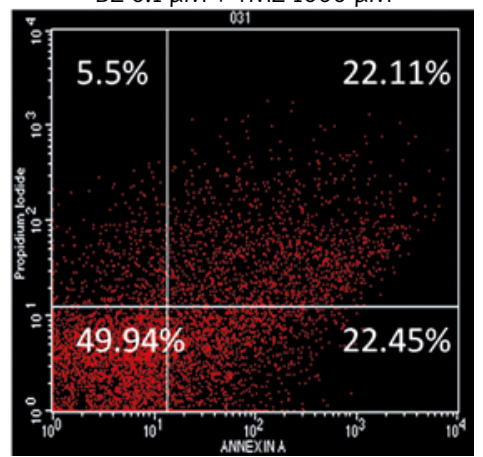

Figure 1. Representative Annexin V/propidium iodide (PI) intensity dot blots of U87MG cells treated for $48 \mathrm{~h}$ with temozolomide (TMZ) and/or bortezomib (BZ) at the indicated doses. Cell death was determined by Annexin V and PI staining (see Material and methods for details). Data are means from two independent experiments run in duplicate $(p<0.01)$

Table I. Synergism quotations of TMZ-BZ combinations in U87 cells

\begin{tabular}{|c|c|c|c|}
\hline \multirow[t]{2}{*}{ Treatment (48 h) } & \multicolumn{3}{|c|}{ Mean \pm standard deviation } \\
\hline & Apoptosis (Annexin+) & Necrosis (PI+) & Both (Annexin+, Pl+, Annexin+/PI+) \\
\hline$T M Z 1 \mu M+B Z 10^{-1} \mu M$ & $0.88 \pm 0.09$ & $0.43 \pm 0.12$ & $0.72 \pm 0.10$ \\
\hline 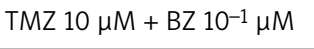 & $0.52 \pm 0.25$ & $0.69 \pm 0.14$ & $0.61 \pm 0.18$ \\
\hline$T M Z 10^{2} \mu M+B Z 10^{-1} \mu M$ & $0.61 \pm 0.23$ & $0.67 \pm 0.17$ & $0.65 \pm 0.21$ \\
\hline
\end{tabular}


tion with $T M Z$ and radiation in different glioma lines, including U87, failed to show radiosensitization of p53 wild-type cells [10]. However, the short time of TMZ exposure ( $3 \mathrm{~h}$ ) and the high sensitivity to NPI-0052 might have compromised the radiotoxic effect of the combinations. In contrast, the NPI-0052-TMZ combination inhibited survival of U87 cells, which is a reproducible effect of proteasome inhibitors when added to TMZ, in our and previous studies [9].

Given the previous isolation and characterization of cancer stem cells from the U87 cell line [12], increased activity of the BZ-TMZ combination might also result from the fact that these drugs target different cell sub-populations. Indeed, TMZ is more toxic for neural stem cells (NSCs) compared to glioma stem cells (GSCs), causing necrotic death at prolonged administration (7 days), whereas BZ induces apoptosis of GSCs, even at doses as low as $5 \mathrm{nM}$, yet produces minimal effects on NSCs [13].

In conclusion, BZ is a potent chemosensitizer in U87 GBM cells during co-administration with $T M Z$, enabling low doses of the latter to be pro-apoptotic. This could theoretically lead to a reduction of hematologic toxicity from protracted courses of TMZ treatment without compromising the anticancer effects of the drug, but needs to be tested in detail in vivo.

\section{Acknowledgments}

The authors would like to thank Professor Aspasia Tsezou, Departments of Cytogenetics and Molecular Genetics, and Biology, University of Thessaly, Faculty of Medicine, 41110 Larissa, and Department of Biomedical Research and Technology, Center for Research and Technology-Thessaly (CE.RE.TE.TH), Larissa 41222, Greece, for use of flow cytometry facilities. We also thank Dr. Athanasios Mavropoulos, Department of Biomedical Research and Technology, Center for Research and Technology-Thessaly (CE.RE.TE.TH), Larissa 41222, Greece, for assistance with flow cytometric analysis.

\section{Conflict of interest}

The authors declare no conflict of interest.

\section{References}

1. Stupp R, Mason WP, van den Bent MJ, et al. Radiotherapy plus concomitant and adjuvant temozolomide for glioblastoma. N Engl J Med 2005; 352: 987-96.

2. Wick W, Platten M, Weller M. New (alternative) temozolomide regimens for the treatment of glioma. Neuro Oncol 2009; 11: 69-79.

3. Quinn JA, Jiang SX, Reardon DA, et al. Phase I trial of temozolomide plus 06-benzylguanine 5-day regimen with recurrent malignant glioma. Neuro Oncol 2009; 11: 556-61.
4. Vlachostergios PJ, Patrikidou A, Daliani DD, Papandreou CN. The ubiquitin-proteasome system in cancer, a major player in DNA repair. Part 1: post-translational regulation. J Cell Mol Med 2009; 13: 3006-18.

5. Vlachostergios PJ, Patrikidou A, Daliani DD, Papandreou CN. The ubiquitin-proteasome system in cancer, a major player in DNA repair. Part 2: transcriptional regulation. J Cell Mol Med 2009; 13: 3019-31.

6. Yin D, Zhou H, Kumagai T, et al. Proteasome inhibitor PS341 causes cell growth arrest and apoptosis in human glioblastoma multiforme (GBM). Oncogene 2005; 24: 344-54.

7. Vlachostergios PJ, Hatzidaki E, Stathakis NE, Koukoulis GK, Papandreou CN. Bortezomib downregulates MGMT expression in T98G glioblastoma cells. Cell Mol Neurobiol 2013; 33: 313-8.

8. Kubicek GJ, Werner-Wasik M, Machtay M, et al. Phase I trial using proteasome inhibitor bortezomib and concurrent temozolomide and radiotherapy for central nervous system malignancies. Int J Radiat Oncol Biol Phys 2009; 74: 433-9.

9. Ng K, Nitta M, Hu L, et al. A small interference RNA screen revealed proteasome inhibition as strategy for glioblastoma therapy. Clin Neurosurg 2009; 56: 107-18.

10. Vlashi E, Mattes M, Lagadec C, et al. Differential effects of the proteasome inhibitor NPI-0052 against glioma cells. Transl Oncol 2010; 3: 50-5.

11. Wang J, Guo J, Wu S, et al. Synergistic effects of nanosecond pulsed electric fields combined with low concentration of gemcitabine on human oral squamous cell carcinoma in vitro. PLoS One 2012; 7: e43213.

12. Yu SC, Ping YF, Yi L, et al. Isolation and characterization of cancer stem cells from a human glioblastoma cell line U87. Cancer Lett 2008; 265: 124-34.

13. Gong X, Schwartz PH, Linskey ME, Bota DA. Neural stem/progenitors and glioma stem-like cells have differential sensitivity to chemotherapy. Neurology 2011; 76: 1126-34. 